\title{
MULTI-SENSOR DATA FUSION FOR LONG RANGE DEMINING AREA REDUCTION
}

\author{
Salvatore Savastano and Raffaella Guida
}

Surrey Space Centre - University of Surrey, Guildford, UK - \{s.savastano, r.guida $\} @$ surrey.ac.uk

\begin{abstract}
Multisensor data fusion is getting more importance with the increasing number of available satellite sensors. The aim of data fusion is to take advantages of combining different types of data to improve accuracies. However features extracted from different sensors will often have different statistical properties, and therefore combining data in an efficient way is not a trivial task. This paper proposes a new algorithm for data fusion between classification maps separately derived by application of clustering algorithms to PolSAR and Multispectral datasets. The expected new output is a map where all the classes identified with each single dataset will be present. The pixels assigned to the same class with both datasets will be characterized by a higher likelihood to belong to that class. The application for which the data fusion has been developed is that of hazardous areas reduction in land mines clearing operation. At this purpose a demonstration site has been set up in Poland within the FP7 D-BOX project and the fusion framework tested on it with acquisition of remote sensing imagery.
\end{abstract}

Index Terms- Multispectral, PolSAR, Clustering, Data fusion, Remote Sensing

\section{INTRODUCTION}

The application of hazardous area reduction via Earth Observation (EO) assets in humanitarian demining of land mines [1] is gaining momentum due also to the high financial cost of demining and clearance activities mandating that patches of land currently in-use (hazard free), inside a suspected hazardous area, do not undergo unnecessary demining and clearance activities.

This paper presents some of the authors most recent contributions to the FP7 Integration Project D-BOX [2], funded by the European Commission with the objective of tackling the issue of anti-personnel landmines and cluster munitions remaining from armed conflicts by developing a comprehensive toolbox for humanitarian clearing of large civil areas. The toolbox is being developed to include several EO capabilities, such as land use and land cover mapping, change detection analysis and can be exploited in the framework of demining activities such as the extraction of specific mineaction-related indicators. The work presented in this paper, representing one of the toolbox capabilities, mainly builds on the need of covering, with EO data, large areas with good detail at a reduced cost with respect to aerial data on limited areas of interest (e.g. minefields usually cover areas smaller than $100 \mathrm{~km}^{2}$ ).

In the last decade, the capability of meeting those needs is greatly improved thanks to the availability of radar and multispectral images provided by the second generation of high resolution satellites (as COSMO-SkyMed and TerraSAR-X for radar data, or GeoEye-1, Worldview-1\&2, Pleiades etc. for multispectral/optical data), which increased strongly the potentiality of EO.Considering the nature of the scenario involved, the direct detection of buried mines is not feasible with modern means and, as anticipated, only indicators of their presence can be detected. A proper use of multi-source EO data is then advised to tackle the problem from different perspectives and finally identify a fusion framework where the potentialities of different EO sensors are brought together to improve the demining area reduction, which is what the authors address here with the data fusion of PolSAR and Multispectral data for a better identification of risk areas boundaries.

\section{METHODOLOGY}

In order to exploit the capabilities of EO data for the identification of indicators supporting hazard area reduction during demining planning phase and post-clearance efficiency monitoring detection, the following reference EO input satellite data have been considered:

- Fully polarimetric SAR data

- Optical /Multispectral data

Fully polarimetric SAR (PolSAR) data have the advantage over single channel data that the electromagnetic scattering information of the target is completely captured. This extra information helps to differentiate targets from clutter more effectively due to the electromagnetic diversity of the received signal. In addition, PolSAR data enables discriminating different dominant electromagnetic scattering mechanisms occurring at the target. PolSAR data have been found to be particularly useful in hazard zone reduction because of their ability to distinguish different scattering mechanisms resulting in a reasonable identification of some of the secondary 
and tertiary indicators of hazard. In this regard, a considerable amount of research has been done in Space and Airborne Mined Area Reduction Tools (SMART) project [3]. A Radarsat-2 (R2) dataset has been tasked and processed for this paper. As Multispectral image, a WordView-2 (WV-2) dataset has been selected for the availability of eight spectral bands at high resolution. WorldView-2 is the first commercial highresolution satellite to provide eight spectral bands in the visible to near-infrared range. Each sensor is narrowly focused on a particular range of the electromagnetic spectrum that is sensitive to a particular feature on the ground, or a property of the atmosphere [4]. For the purpose of landmine area reduction, PolSAR and Multispectral data are jointly used to improve the detection of some indicators of mine presence/absence as shown in the flowchart in Figure 1. The whole process includes the following steps:

- if the Multispectral data are partially covered or completely clouds-free they enter the Automatic Clustering block together with the PolSAR data, otherwise only PolSAR data are considered but this case is not discussed here given the nature of the paper; here, the two datasets, acquired on the same area and with the shortest possible time delay, are separately used with two different unsupervised algorithms, previously designed and developed [5] [6], to produce clustering maps;

- the two clustering maps are converted, thanks to ancillary information related to land use and morphology, database of mine and demining accidents etc., in classification maps where classes are related to indicators highlighted by end-users. The same list of indicators is applied to both datasets but, given the different source of the datasets, they might not necessarily contain the same number/type of classes;

- once the classified maps have been produced, they are georeferenced in order to have the integrity of a geographic map (the geodetic reference system WGS-84 has been adopted here) and can be optionally visualized on Google Earth for a better understanding and easy use from non-experts;

- the two georeferenced classification maps finally enter the data fusion block where, on the whole area of interest, a simple decision rule is initially applied:

\footnotetext{
if the single pixel has been identified as class $A$ with both datasets and clustering algorithms then return class $\mathrm{A}$ in the output image; else

keep both classes derived by the two datasets and assign a weight/likelihood to each of them; end
}

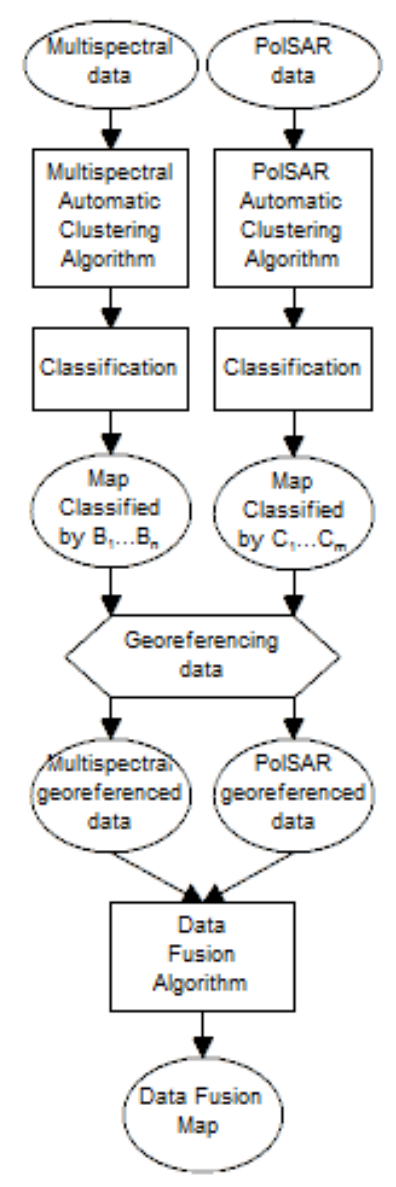

Fig. 1. Flowchart of the data fusion tool

The weight is assigned considering and exploiting the fields of application in which, from literature, each dataset usually outperforms the other as well as the acquisition time of each dataset (with the closest considered more reliable).

Going more into detail in the description of the clustering algorithms, for the PolSAR data the algorithm developed in [5] is adopted. This algorithm is based on modeling the PolSAR data using a certain non-Gaussian statistical distribution. The algorithm determines as many statistically distinct clusters from the PolSAR image as possible. It stops when all the distinct image clusters pass their statistical goodnessof-fit test to the underlying non-Gaussian probability distribution. During the course of its lifetime, the algorithm splits poorly fitted clusters and merges similar clusters till either all clusters are good fits or the maximum number of iterations is reached. The output of the algorithm is a cluster map, depicting the statistically distinct clusters present in the PolSAR image in different colors.

For the Multispectral data, instead, the algorithm developed in [6] for WV-2 is applied. This is effectively an adaptation of the previously mentioned algorithm, and thus automat- 


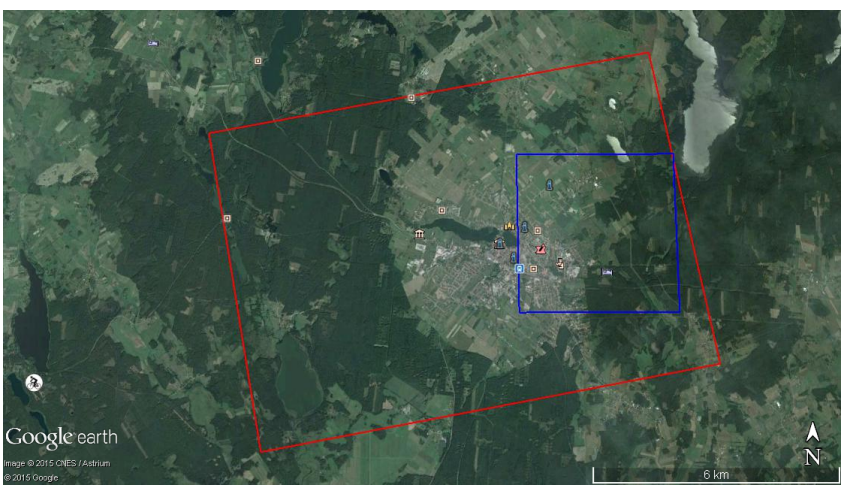

Fig. 2. Area of case study in Szczytno (Poland) with the R2 footprint in red and the WV-2 footprint in blue.

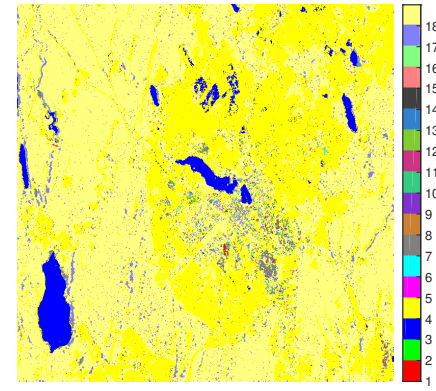

(a)

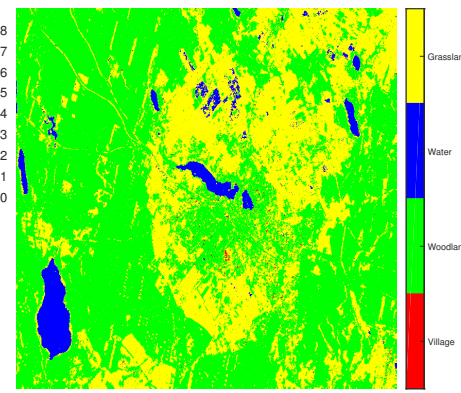

(b)
Fig. 3. Clustering (a) and classification (b) maps from PolSAR data on the area in red frame in Fig.2.

ically determines the number of statistically distinct clusters in the WV-2 multispectral data. As anticipated, the unsupervised clustering algorithms either require the number of clusters to be defined beforehand, or cluster the image at many different hierarchical levels after which a certain threshold has to be defined to select the appropriate level of clustering. In either case, the end result is strongly dependent on the number of clusters or some other threshold initially specified. In both cases, the clusters are semantically unnamed but each of them can identify certain indicators of mine presence/absence (e.g. bridges, river banks, shores of ponds, agricultural fields, tracks, edges of forests etc.). The categorization of clusters in classes with a semantic meaning then follows to derive two distinct classification maps. A trained operator will perform this classification on the base of the ground truth available [7] or, in absence of this, with the help of optical images which, of course, might not have been acquired concurrently with the dataset used and can then affect the classification quality.

\section{DEMONSTRATION}

A demonstration has been prepared within the D-BOX project to assess, among other things, the effectiveness of the clustering algorithms in a data fusion context. A site has been

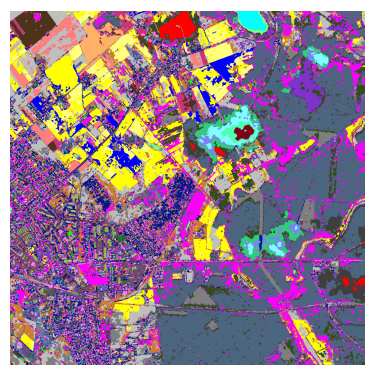

(a)

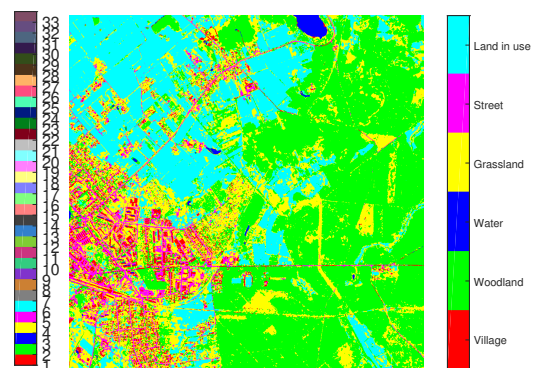

(b)
Fig. 4. Clustering (a) and classification (b) maps from PolSAR data on the area in red frame in Fig.2.

selected in Szczytno (Poland) and it has been prepared to contain or develop several minefield indicators that the tool here presented may detect. The modifications brought to the original site include buried mine shells and fences above all. An acquisition of both R2 and WV-2 datasets has been tasked with the shortest possible temporal gap between the two. The R2 dataset has been acquired on $3^{\text {rd }}$ May 2015, about 6 months after the site was prepared in order to allow vegetation to grow. The $\mathrm{WV}-2$ was tasked with a max value of $15 \%$ for cloud coverage which dictated an acquisition temporal window of 1.5 to 3 months not yet closed at the time of writing. For these reasons, an archive WV-2 dataset from $9^{\text {th }}$ May 2012 is used in this paper to show clustering and classification maps on the selected site for both datasets while the $2015 \mathrm{WV}-2$ dataset will be used for presentation at the conference.

\section{RESULTS}

In Figs. 3 - 4 the output classification maps are shown, respectively, for PolSAR data and Multispectral. Their goodness by visual inspection is commented in the following while, as anticipated, fusion of maps based on the simple decision rule in Section 2, will be performed directly on the WV-2 dataset tasked for acquisition in June-July 2015 and final results presented at the conference.

\subsection{PolSAR data}

After preprocessing, necessary to reduce speckle noise typical of SAR images, the PolSAR data presents a resolution of $10 \mathrm{~m} \times 10 \mathrm{~m}$ roughly. At this resolution the clustering it is not able to distinguish specific indicators proposed by D-Box end users such as signs of vehicle, presence of fences, etc. Despite of that, the clustering map in Fig. 3(a) still allows the detection of the following features:

- shallow water in areas of grassland, probably due to paddy fields or recently watered fields (maybe flooded if in proximity of water bodies); 
- narrow and long lines inside a woodland, possibly due to routes or rail tracks;

- borders between forested areas and grassland;

- streets (but if the number of classes is small they may be associated to a wrong class - grassland in the example classification map in Fig. 3(b)).

Isolated buildings, especially if separated by vegetation, are not detected due also to the poor resolution $(10 \mathrm{~m} \times 10 \mathrm{~m})$. This is, for example, the case of the demonstration test site (not shown in the above pictures). Only the land around is rightly classified as grassland or forests but the resolution does not allow any distinction among test sites.

\subsection{Multispectral data}

Thankful to a better resolution ( $2 \mathrm{~m} \times 2 \mathrm{~m})$ the clustering algorithm for multispectral data is able to perform a finer clustering, Fig. 4(a) and, in turn, classification becomes more accurate with more classes identified, Fig. 4(b). The map classified permits to reduce hazardous area identifying:

- generic grassland, divided by land in use, as agriculture fields;

- streets inside village but also inside a woodland;

- narrow routes;

- borders between forested areas and grassland;

- buildings;

- water bodies.

Being the WV-2 image from archive it has not been possible to identify several minefield indicators such as signs of vehicle, presence of fences, etc, proposed by D-Box end users and placed on the site of demonstration. However this test will be performed when the new WV-2 data will be received together with the final fusion of the maps.

\section{CONCLUSIONS}

A data fusion algorithm for PolSAR and Multispectral data, based on existing clustering algorithms for the original data, has here been proposed. The fusion suggested is at thematic level and simple decision rules have been introduced in order to allow its application from non expert, but trained, users. Similarly, classification has been performed having the same end users in mind, identifying just the number and kind of classes they would probably recognize using, in a worst case scenario, outdated optical images as ground truth. Due to the fact that the temporal window for the WV-2 acquisition is not yet closed at the time the authors write, final test on fusion has not been performed here but will be presented at the conference.

\section{ACKNOWLEDGEMENTS}

Research carried out for this paper has been funded by the European Commission within the FP7 project D-BOX: Demining tool-BOX for humanitarian clearing of large scale areas from anti-personnel landmines and cluster munitions.

\section{REFERENCES}

[1] Yong Yu, I. Bloch, and A. Trouve, "A unified unsupervised clustering algorithm and its first application to landcover classification," in Acoustics, Speech, and Signal Processing, 2003. Proceedings. (ICASSP '03). 2003 IEEE International Conference on, 2003, vol. 3, pp. III689-92.

[2] "Demining toolbox (D-Box), " http://www.dboxproject.eu/, 2003, [Online; accessed 5-December2015].

[3] M. Acheroy, "Smart: Space and airborne mined area reduction tools" http://www.smart.rma.ac.be/, November 2008, [Online; accessed 5-December-2015].

[4] "The benefits of the 8 spectral bands of worldview-2," https://www.digitalglobe.com, March 2010, [Online; accessed 5-December-2015].

[5] Salman Khan and Anthony Paul Doulgeris, "Unsupervised clustering of polsar data using polarimetric $\mathrm{g}$ distribution and markov random fields," in Proc. EUSAR 2014, Berlin, Germany, 03-05 June 2014, pp. 1025-1028.

[6] S. Khan, A.P. Doulgeris, S. Savastano, and R. Guida, "Automatic clustering of multispectral data using a nongaussian statistical mode" in Proc. IGARSS 2014, Quebec, Canada, 13-18 July 2014, pp. 4276-4279.

[7] "Hazard zone reduction via space assets and airborne assets," Tech. Rep., D-BOX PROJECT WP5, 2013, FP7 D-BOX Internal report. 